\title{
A Contact Force Control System Distributed over Heterogeneous Networks
}

\author{
Nayeon Kim and Chanwoo Moon \\ School of Electrical Engineering, Kookmin Univ. \\ mcwnt@kookmin.ac.kr
}

\begin{abstract}
For decades, the use of electronic control units (ECUs) in vehicles has been steadily increasing. The ECUs are connected to in-vehicle networks, for which CANs (Controller Area Networks) are widely used and FlexRay has recently emerged. In this paper, a contact force control system that is distributed over a CAN and FlexRay system is presented. To transfer data within the allowed delay time with limited Frame IDs, a packing algorithm that allows multiplexing of a FlexRay slot is proposed and an Integer Program problem is formulated to build a packing map. A network-based control system is implemented and a contact force control experiment is conducted which verifies the proposed method.
\end{abstract}

Keywords: Interconnected network, Network based control, CAN, FlexRay, Actuator control

\section{Introduction}

For decades, many of the main control parts in vehicles have been converted from mechanical to electronic systems. Accordingly, the number of electronic control units (ECUs) has increased and the need for high speed in-vehicle networks (IVNs) has emerged to process the large amount of data passed among the ECUs. IVNs include LIN (Local Interconnect Network), CAN (Controller Area Network), FlexRay, and MOST (Media-Oriented Systems Transport). MOST is used to connect multimedia devices such as audio and amplifiers in vehicles, while for control purposes, CAN and FlexRay are used.

The CAN developed by Bosch in the 1980s has been widely used as a control network system for vehicle bodies and chassis [1]. It is an event-triggered protocol and can send up to 8 bytes per message at 1 Mbps. Furthermore, a CAN-based system is easy and cheap to implement, and has an internal fault detection function, so is considered to be a reliable protocol and suitable for control applications. However, its transmission speed is unsatisfactory for large amounts of data, thus the need for a more complex and fast network structure as well as the application of X-by-wire technology has been recognized in the vehicle industry. For this reason, the FlexRay consortium announced the FlexRay protocol for in-vehicle networking [2] based on both time trigger and event trigger protocols, and can realize transmission speeds of up to $10 \mathrm{Mbps}$ while running on simple hardware. However, since the FlexRay protocol can only communicate after all of the messages have been scheduled by the network designer, it is time-consuming to design and expensive.

Since CAN is reasonable for implementing a network for low cost devices, it is efficient solution to use CAN and FlexRay in combination. Low cost devices are connected to CAN, while devices that require real-time control are connected to FlexRay. In this case, the gateway performs FlexRay-CAN protocol conversion. In their respective

Received (May 25, 2017), Review Result (October 18, 2017), Accepted (October 20, 2017) 
studies, Shaheen and et al. [3] implemented a FlexRay-CAN gateway and Seo et al. [4] and $\mathrm{Li}$ et al. [5] proposed a transformation map between a CAN message and a FlexRay message, and confirmed that the message was converted correctly through experiments. Schmidt [6] implemented a gateway considering the Worst Case Response Time (WCRT) between the CAN and FlexRay nodes but did not mention a packing problem of the CAN signals. Nevertheless, in a real-time control system, a transfer delay can affect system stability $[7,8]$.

In this paper, we present a contact force control system in which the sensor and actuator are distributed over the CAN and FlexRay network. To transfer the sensor signals of a CAN node to an actuator node on the FlexRay network within the deadline, a new packing method allowing multiplexing of FlexRay message slots is presented. To build a packing map, we formulate an ILP (Integer Linear Programming) problem and implement a gateway with the proposed packing method, in which a limited number of Frame IDs were assigned. Finally, an experiment is conducted to evaluate the proposed packing method.

\section{Structure of a Contact Force Control System}

\subsection{Construction of a Control Network}

FlexRay is a high speed time-triggered system that fulfils the role of a network backbone, and low cost sensors and devices are connected to a CAN. FlexRay and CAN data are transmitted from to the FlexRay network via a gateway. Figure 1 shows the network structure of a contact control system. A force sensor with a strain gauge and other sensors are connected to the CAN, while a real-time controller for the actuator is connected to the FlexRay network, and periodically, data are transferred along the static segment of the FlexRay frame. Sensor data are transmitted to the FlexRay-CAN gateway via the CAN, packed into a FlexRay packet, and transferred to the controller.

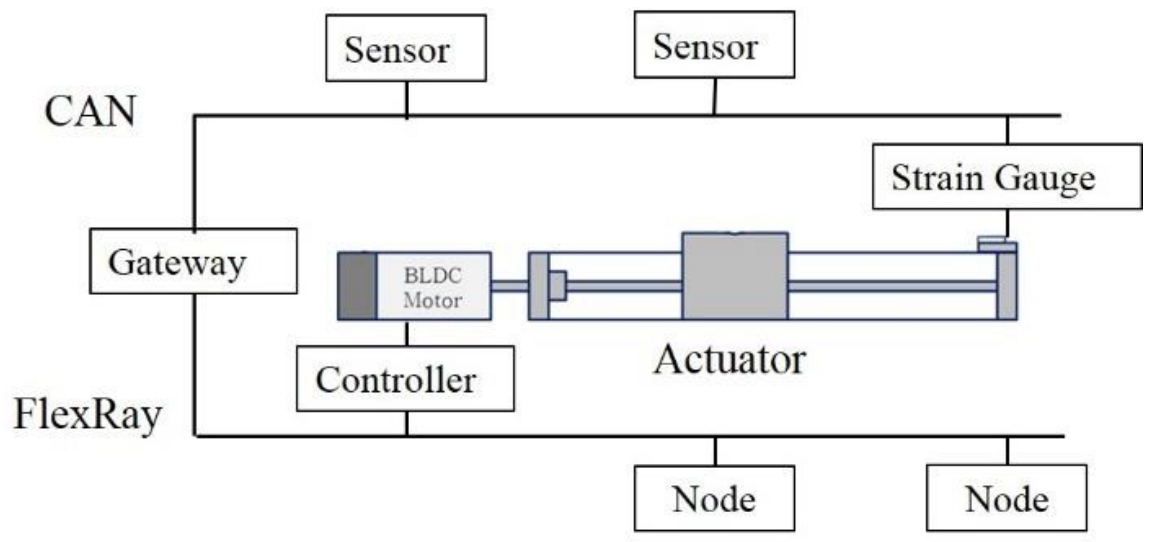

Figure 1. Network Structure of the Contact Force Control System

\subsection{Structure of Control System}

A simulation block diagram of the whole contact force control system is shown in Figure 2. The actuator consists of a brushless DC (BLDC) motor, a linear stage, and a strain gauge to measure the contact force. The transfer function of the system excluding transmission delay is given by

$$
\mathrm{H}(\mathrm{s})=\frac{k}{M s^{2}+b s+k},
$$

where $\mathrm{M}$ is the mass of the moving part, $\mathrm{b}$ is the damping coefficient, and $\mathrm{k}$ is the strain gauge stiffness[9]. The controller uses a proportional integral (PI) control rule, and 
the torque command from the controller is sent to the BLDC current controller. The Simulink simulation results show that the control system became unstable when the transmission delay exceeded $50 \mathrm{~ms}$. Considering the stability margin and jitter at the controller, we set the allowed time delay to $10 \mathrm{~ms}$.

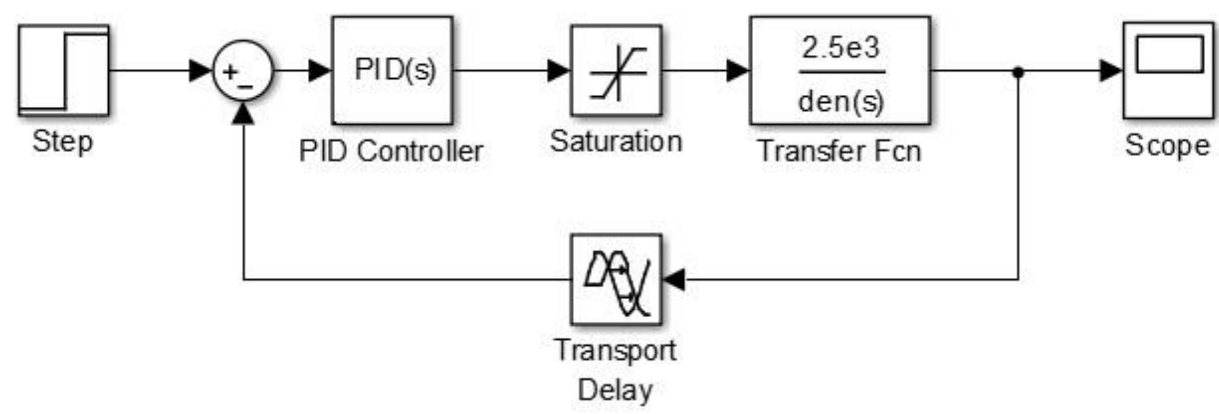

(a) Simulation Block
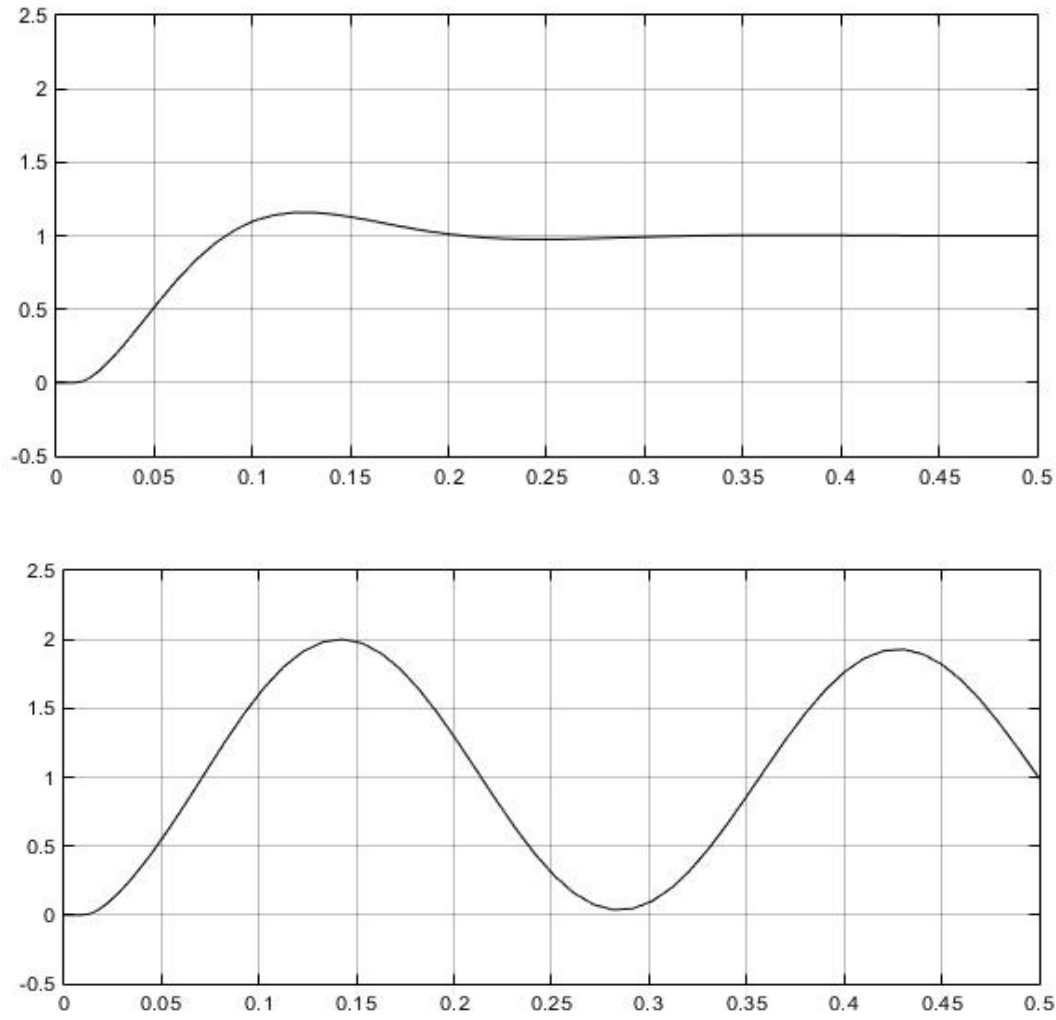

(b) Step Response with $10 \mathrm{~ms}$ (upper graph) and $50 \mathrm{~ms}$ (lower graph) Delay

Figure 2. Simulation Block and Step Response of the Contact Force Control System

\section{Design of a Gateway}

\subsection{Worst Case Response Time for Stability}

One cycle of FlexRay consists of static segment (SS), dynamic segment(DS), symbol window(SW), and network idle time(NIT) [2]. The static segment and network idle time are indispensable and the dynamic segment and symbol window are optional. The static segment uses the TDMA (Time Division Multiple Access) scheme, and includes a number of static slots, which have their unique frame IDs. So, the static segment is 
suitable for periodic control and sensor messages, and in this paper, only the static segment is considered for control purpose. The dynamic segment uses the FTDMA (Flexible Time Division Multiple Access) scheme.

In order to maintain the stability of a network-based control system, the worst case response time (WCRT) of a message on the network must be less than the allowed time delay. The total WCRT $\mathrm{Rs}_{\mathrm{n} \text { (Total) }}$ that it takes for signal $\mathrm{s}_{\mathrm{n}}$ from a sensor node to arrive at the controller after transmission is calculated as follows [10].

$$
\mathrm{R} s_{n(\text { Total })}=\mathrm{R} s_{n(\text { FlexRay })}+\mathrm{R} s_{n(C A N)}
$$

where $\mathrm{R} s_{n \text { (FlexRay) }}$ is the WCRT on the FlexRay network, $\mathrm{R} s_{n(C A N)}$ is the WCRT on the CAN. CAN is an event triggered communication protocol, so collision between messages can occur. Network transmission delay is stochastically varying within the range of the WCRT and the $\mathrm{Rs}_{\mathrm{n}(\mathrm{CAN})}$ of signal $\mathrm{s}_{\mathrm{n}}$ is obtained as equation (3) [11].

$$
\mathrm{R} s_{n(C A N)}=J_{s}+\omega_{s}-q T_{s}+C_{s}
$$

where $J_{S}$ is the jitter, $\omega_{S}$ is the queuing delay, $T_{S}$ is the minimum inter-arrival time, $q$ is the message instance, and $\mathrm{C}_{\mathrm{S}}$ is the signal communication time. Since the data size of FlexRay is usually larger than the data size of CAN, several CAN signals are packed into one FlexRay message as shown in Figure 3 (a) [4]. Each FlexRay message has a frame ID (FID), repetition cycle, and cycle offset. The allowed repetition cycles in the FlexRay standard are $\{1,2,4,5,8,10,16,20,32,40,50,64\}$. A slot can be multiplexed using multiple messages with a long repetition cycle, as shown in Figure 3 (b). The longer the repetition cycles that multiplexed messages have, the more data can be sent via one message slot and the more the WCRT increases. Subsequently, in this study, only the bisection of one slot was allowed; that is to say, messages sent via the gateway had repetition cycles $\{1,2,4,8,16,32,64\}$, and so one slot was shared by $1,2,4,8,32$, or 64 messages.

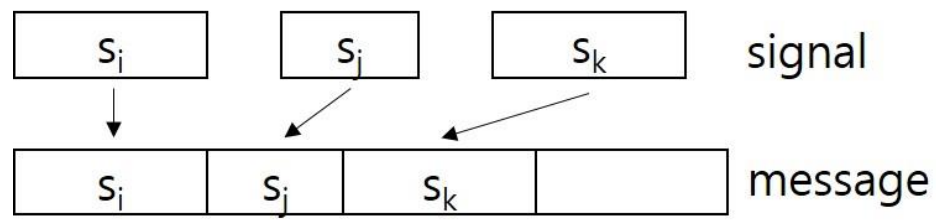

(a) Packing of Signals Into Message

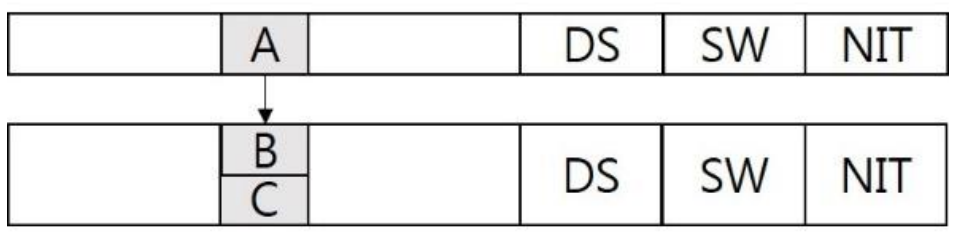

(b) Multiplexing of a Flexray Slot

\section{Figure 3. Packing and Multiplexing of FlexRay Message}

The FlexRay gateway is part of whole FlexRay network, and the global parameters related to the gateway should be set equal to the parameters of the entire network. The parameters that can be designed in the gateway are the FID number, repetition cycle, and cycle offset of the message as well as the packing method. Suppose that the global parameters of the FlexRay network are as follows:

V: the size of a FlexRay message (Bytes, $=2 \cdot \mathrm{gPayloadLengthStatic}$ ), $\mathrm{M}_{\text {cycle: }}$ Maximum cycle counter value in a given cluster (=gCycleCountMax).

Definition 1: Message group $\mathrm{C}_{\mathrm{k}}\left(\mathrm{C}_{\mathrm{k}}=1,2, \ldots, \mathrm{K}_{\mathrm{c}}\right)$ 
By bisecting a slot, if a message is sent every $2^{\left(C_{k}-1\right)}$ cycles, it forms the message group $\mathrm{C}_{\mathrm{k}}$, where the maximum number $\mathrm{K}_{\mathrm{c}}$ is an integer satisfying the following conditions:

$$
\left(\mathrm{M}_{\text {cycle }}+1\right) / 2^{\left(K_{c}-2\right)} \geq 2, \text { and }\left(\mathrm{M}_{\text {cycle }}+1\right) / 2^{\left(K_{c}-1\right)}<2 .
$$

A message belonging to group $\mathrm{C}_{\mathrm{k}}$ is sent $\left[\left(M_{\text {cycle }}+1\right) / 2^{K_{c}-1}\right\rfloor$ times in $\left(\mathrm{M}_{\text {cycle }}+1\right)$ cycles, and the WCRT for this message on FlexRay network, $\operatorname{R} s_{n(\text { FlexRay) }}\left(C_{k}\right)$, is obtained as follows:

- $\quad$ If $\left(\mathrm{M}_{\text {cycle }}+1\right) \mathrm{MOD}$ repetition_cycle $=0$

$$
\mathrm{Rs}_{n(\text { FlexRay })}\left(C_{k}\right)=2^{C_{k}-1} C_{\text {cycle }}+C_{n}
$$

- $\quad$ Else, that is $\left(\mathrm{M}_{\text {cycle }}+1\right)$ MOD repetition_cycle $\neq 0$

- If $0 \leq$ cycle offset of the message $\leq\left\{\left(\mathrm{M}_{\text {cycle }}+1\right)\right.$ MOD repetition_cycle -1$\}$

$$
\mathrm{Rs}_{n(\text { FlexRay })}\left(C_{k}\right)=2^{C_{k}-1} C_{\text {cycle }}+C_{n}
$$

- $\quad$ Else

$$
\begin{aligned}
& \mathrm{R} s_{n(\text { FlexRay })}\left(C_{k}\right)=\left\{2^{C_{k}-1}+\left(M_{\text {cycle }}+1\right) M O D\right. \\
& \text { repetition cycle }\} C_{\text {cycle }}+C_{n}
\end{aligned}
$$

where $\mathrm{C}_{\text {cycle }}$ is the FlexRay 1 Cycle period and $\mathrm{C}_{\mathrm{n}}$ is the message transmission time. From equations (4)-(6), $R s_{n \text { (FlexRay) }}\left(C_{k}\right)$ can be written as

$$
\begin{gathered}
\mathrm{R}_{n(\text { FlexRay })}\left(C_{k}\right)=\left\{\begin{array}{c}
\left.2^{C_{k}-1}+\left(M_{\text {cycle }}+1\right) \text { MOD repetition cycle }\right\} C_{\text {cycle }}+C_{n}, \\
\left(\mathrm{C}_{\mathrm{k}}=1 \ldots \mathrm{K}_{\mathrm{c}}-1\right)
\end{array}\right. \\
\mathrm{R} s_{n(\text { FlexRay })}\left(C_{k}\right)=\left(M_{\text {cycle }}+1\right) C_{c y c l e}+C_{n},\left(\mathrm{C}_{\mathrm{k}}=\mathrm{K}_{\mathrm{c}}\right)
\end{gathered}
$$

Let $d_{\mathrm{sn}}$ denote the whole allowed delay of signal $s_{n}$, then the allowed delay on FlexRay network, $d_{\text {sn(FlexRay) }}$, is derived as

$$
d_{\left.s_{n} \text { (FlexRay }\right)}=d_{s n}-R_{S_{n}(\text { CAN })} \text {. }
$$

Definition 2: Signal group $b_{\mathrm{n}} \in 1,2, \ldots, K_{c}$

Signal $\mathrm{s}_{\mathrm{n}}$ forms signal group $\mathrm{b}_{\mathrm{n}}$

$$
\begin{gathered}
\text { If } R_{\left.s_{n} \text { (FlexRay }\right)}\left(b_{n}\right) \leq d_{\left.s_{n} \text { (FlexRay }\right)}<R_{\left.s_{n} \text { (FlexRay }\right)}\left(b_{n}+1\right) \\
\text { And } R_{\left.S_{n} \text { (FlexRay }\right)}\left(K_{c}+1\right)=\infty \text {. }
\end{gathered}
$$

If $b_{n} \geq C_{k}$, then message group $C_{\mathrm{k}}$ is said to be higher than or equal to signal group $b_{\mathrm{n}}$. The gateway packs the signals from the CAN into messages and sends them to the FlexRay network inside the deadline with the assigned finite number of FIDs. The packing principles are as follows:

- To minimize the number of FID used in gateway, signals are packed before sending.

- $\quad$ So as not to exceed the deadline, all signals are packed into higher or equal message group.

- $\quad$ The gateway multiplexes one slot by cycles in order to send all messages.

- The number of messages should be minimized and must not exceed the number of message boxes that the FlexRay communication controller has allocated for the transmission.

To obtain a packing map satisfying the packing principle, an Integer Linear Programming problem was formulated with the following notation:

$\mathrm{N}_{\mathrm{F}}$ : the number of FIDs assigned to the gateway

$\mathrm{N}_{\mathrm{M}}$ : the number of messages for each FID

$\mathrm{N}_{\text {MMAX: }}$ the number of message boxes for transmission

$\mathrm{N}_{\mathrm{S}}$ : the number of CAN signals to send

$F_{\mathrm{i}}:$ FID, $\quad 1 \leq \mathrm{i} \leq \mathrm{N}_{\mathrm{F}}$ 
$\mathrm{m}_{\mathrm{ij}}$ : message $\mathrm{j}$ of FID $\mathrm{I}, \quad 1 \leq \mathrm{j} \leq \mathrm{N}_{\mathrm{M}}$

$\mathrm{s}_{\mathrm{n}}$ : signal, $1 \leq \mathrm{n} \leq \mathrm{N}_{\mathrm{S}}$

$a_{n}$ : data size of signal $s_{n}$ (Bytes)

$\mathrm{y}_{\mathrm{ijk}}=1$ if Message $\mathrm{j}$ of FID $\mathrm{i}$ is in message group $\mathrm{C}_{\mathrm{k}}$, else 0

$\mathrm{z}_{\mathrm{ijn}}=1$ if signal $\mathrm{s}_{\mathrm{n}}$ is sent on Message $\mathrm{j}$ of FID $\mathrm{i}$, else 0

$$
\mathrm{x}=\left(\begin{array}{l}
y \\
z
\end{array}\right)
$$

$f_{C_{k}}$ : the fraction of a $C_{k}$ group message in one FID slot $\left(f_{C_{k}} \in\left\{f_{1}, f_{2}, \ldots, f_{K_{C}}\right\}\right)$

The integer programming [12] formula for this packing problem is as follows:

Object :

Minimize the number of FIDs used

$$
\min _{x} f^{T} x, \quad f^{T}=\left\{f_{1}, f_{2}, \ldots f_{K_{c}}, f_{1}, \ldots f_{K_{c}}, \ldots f_{1}, \ldots f_{K_{c}}, 0,0, \ldots 0\right\}
$$

Equality constraints:

(1) All CAN signals must be included in a FlexRay message.

For all $\mathrm{n}, \sum_{i} \sum_{j} z_{i j n}=1$

Inequality constraints:

(1) Messages belonging to FID i must be transmitted with FID i.

For all i, $\sum_{j} \sum_{k} 2^{-(k-1)} y_{i j k} \leq 1$

(2) The size of the data included in the message $\mathrm{j}$ of the FID $\mathrm{i}$ must be equal to or smaller than V.

For all i,j, $\sum_{n} a_{n} z_{i j n} \leq V \sum_{k} y_{i j k}$

(3) All signals must be transferred with higher or equal message group.

For all $\mathrm{i}, \mathrm{j}, \mathrm{n}, b_{n} z_{i j n} \leq \sum_{k}\left(K_{c}-k+1\right) y_{i j k}$

(4) Each message $m_{i j}$ must be contained in at most one message group.

For all i,j, $\sum_{k} y_{i j k} \leq 1$

(5) The number of messages used for transmission should be limited to $\mathrm{N}_{\text {MMAX. }}$.

$\sum_{i} \sum_{j} \sum_{k} y_{i j k} \leq N_{M M A X}$

\section{The Contact Force Control Experiment}

The FlexRay parameters used in the experiment were as follows: $\mathrm{V}=20$ bytes, transmission speed $=10 \mathrm{Mbit} / \mathrm{s}, 1$ cycle $=5 \mathrm{~ms}$, and Mcycle $=63$. In this case, the WCRT and deadline ranges for each message groups were as shown in Table 1. Messages in Message Group 1 can be delivered in $5.06 \mathrm{~ms}$ in the worst case and the signals which has the deadline between $5.06 \mathrm{~ms}$ and $10.06 \mathrm{~ms}$ must be packed into a Message Group 1 . Non-critical signals which has deadline longer than $320.06 \mathrm{~ms}$ can be packed into any Message Group.

Table 1. WCRT and Deadline Range for each Message Group of FlexRay

\begin{tabular}{|c|c|c|c|}
\hline $\begin{array}{c}\text { Repetition } \\
\text { Cycle }\end{array}$ & Message Group & $\begin{array}{c}\mathrm{Rs}_{\text {(FexRay) }} \\
(\mathrm{ms})\end{array}$ & $\begin{array}{c}\text { Deadline range } \\
(\mathrm{ms})\end{array}$ \\
\hline 1 & 1 & 5.06 & $5.06 \sim 10.06$ \\
2 & 2 & 10.06 & $10.06 \sim 20.06$ \\
4 & 3 & 20.06 & $20.06 \sim 40.06$ \\
8 & 4 & 40.06 & $40.06 \sim 80.06$ \\
16 & 5 & 80.06 & $80.06 \sim 160.06$ \\
32 & 6 & 160.06 & $160.06 \sim 320.06$ \\
64 & 7 & 320.06 & $320.06 \sim$ \\
\hline
\end{tabular}


The total number of CAN signals was 16 and among them, 12 signals were transferred to the FlexRay network. The CAN WCRT and signal group for each signal are as shown in Table 2 for when the transmission speed of CAN was $1 \mathrm{Mbit} / \mathrm{s}$. The force sensor data was assigned to signal 5. After solving the ILP with 2 FIDs, no solution could be found. With 3 FIDs, a packing solution was obtained, as shown in Table 3. FID 1 used 3 messages with repetition cycle 2,4 , and 4 , and signals $4,8,9,10,11$, and 12 were transferred with FID 1. FID 2 and 3 used one message of repeat cycles 2 and 1, respectively. Signals 5, 6 and 7 were packed in FID 2 message and signals 1,2 and 3 were packed in FID 3. The proportion of the fraction occupied by the messages in each FID is $1.0,0.5$ and 1.0 respectively. FID 1 and 3 are fully used and FID 2 has room for further message transfer.

Table 2. Deadline, CAN WCRT and Signal Group of each CAN Signal

\begin{tabular}{|c|c|c|c|c|c|}
\hline $\begin{array}{c}\text { Signal No. } \\
\text { (Priority) }\end{array}$ & $\begin{array}{c}\text { Period } \\
(\mathrm{ms})\end{array}$ & $\begin{array}{c}\text { Data Size } \\
(\text { Byte })\end{array}$ & $\begin{array}{c}\text { Total } \\
\text { Deadline } \\
(\mathrm{ms})\end{array}$ & $\begin{array}{c}\text { CAN } \\
\text { WCRT } \\
(\mathrm{ms})\end{array}$ & $\begin{array}{c}\text { Signal } \\
\text { Group }\end{array}$ \\
\hline $1(1)$ & 8 & 8 & 12 & 1.32 & 2 \\
$2(2)$ & 5 & 6 & 10 & 1.46 & 1 \\
$3(5)$ & 10 & 8 & 14 & 1.92 & 2 \\
$4(8)$ & 5 & 5 & 10 & 2.35 & 1 \\
$5(6)$ & 5 & 8 & 10 & 2.08 & 1 \\
$6(9)$ & 10 & 4 & 14 & 2.47 & 2 \\
$7(10)$ & 12 & 6 & 14 & 2.61 & 2 \\
$8(11)$ & 20 & 7 & 18 & 2.76 & 2 \\
$9(13)$ & 30 & 8 & 25 & 3.08 & 3 \\
$10(14)$ & 40 & 8 & 26 & 3.24 & 3 \\
$11(15)$ & 50 & 8 & 46 & 3.38 & 4 \\
$12(16)$ & 100 & 6 & 56 & 3.38 & 4 \\
\hline
\end{tabular}

Table 3. Packing Map

\begin{tabular}{|c|c|c|c|c|}
\hline \multirow{2}{*}{ FID } & Message & $\begin{array}{c}\text { Repeat } \\
\text { Cycle }\end{array}$ & $\begin{array}{c}\text { Packed } \\
\text { Signal } \\
\text { No. }\end{array}$ & $\begin{array}{c}\text { Used Data Size } \\
\text { (Byte) }\end{array}$ \\
\hline \multirow{3}{*}{1} & 1 & 4 & 11,12 & 14 \\
\cline { 2 - 5 } & 2 & 2 & 4,8 & 15 \\
\cline { 2 - 5 } & 3 & 4 & 9,10 & 16 \\
\hline 2 & 1 & 2 & $5,6,7$ & 18 \\
\hline 3 & 1 & 1 & $1,2,3$ & 19 \\
\hline
\end{tabular}

Figure 4 shows the whole control system and gateway. The FlexRay-CAN gate way and FlexRay node were implemented with Infineon 32bit TC1782 board and the CAN nodes were implemented with Infineon 16bit XC2287 board. The contact force controller were implemented on the FlexRay node and the contact force sensor data were measured 
by a CAN node. Figure 5 depicts the experimental results with step command input. The step command was applied with a value of $1 \mathrm{~N}$ at $25 \mathrm{~ms}$. And it shows that the control system operated stably even with a $10 \mathrm{~ms}$ time delay of sensor data. Excessive overshoot which did not appear in the Matlab simulation may be explained due to sensor assembly tolerances.

\section{Conclusions}

In this paper, a contact force control system that has distributed sensors and an actuator on a CAN and FlexRay interconnected network is presented. Distributed low cost sensors were connected to the CAN, while the real-time controller was connected to the FlexRay network. The implemented controller consisted of a BLDC motor, a linear stage, and a force sensor. In order to maintain the stability of the force control system, the WCRT of a message on the network must be less than the allowed time delay. The allowed time delay was obtained through Matlab simulation, and a gateway to interconnect the CAN and FlexRay systems was implemented. A packing algorithm that transferred the CAN signals within the allowed delay time with a limited number of FIDs was constructed and an Integer Linear Programming problem was formulated to build the packing map. The object of the ILP was to minimize the used fraction of the FIDs when a limited number of FIDs were given under the constraints that the sensor signals of a CAN node must be transfer to an actuator node on the FlexRay network within the deadline. A contact force control experiment was conducted to verify the proposed method and showed that the gateway and control system maintained stability despite the transmission time delay. The proposed method could be useful in the design of interconnected and distributed networkbased control systems.

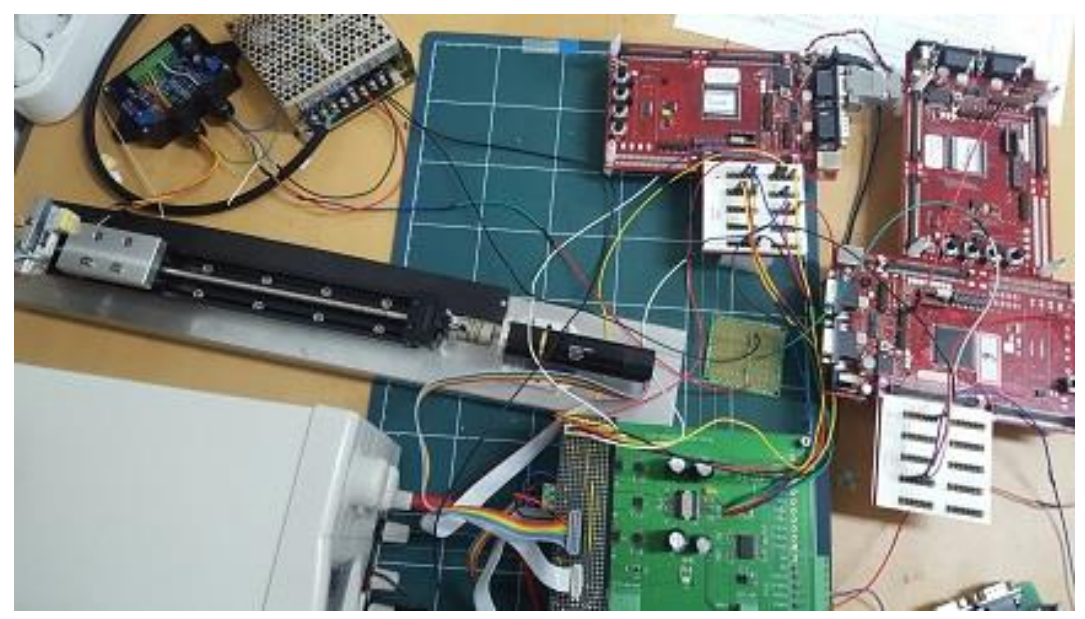

Figure 4. Pressure Control Actuator and Gateway 


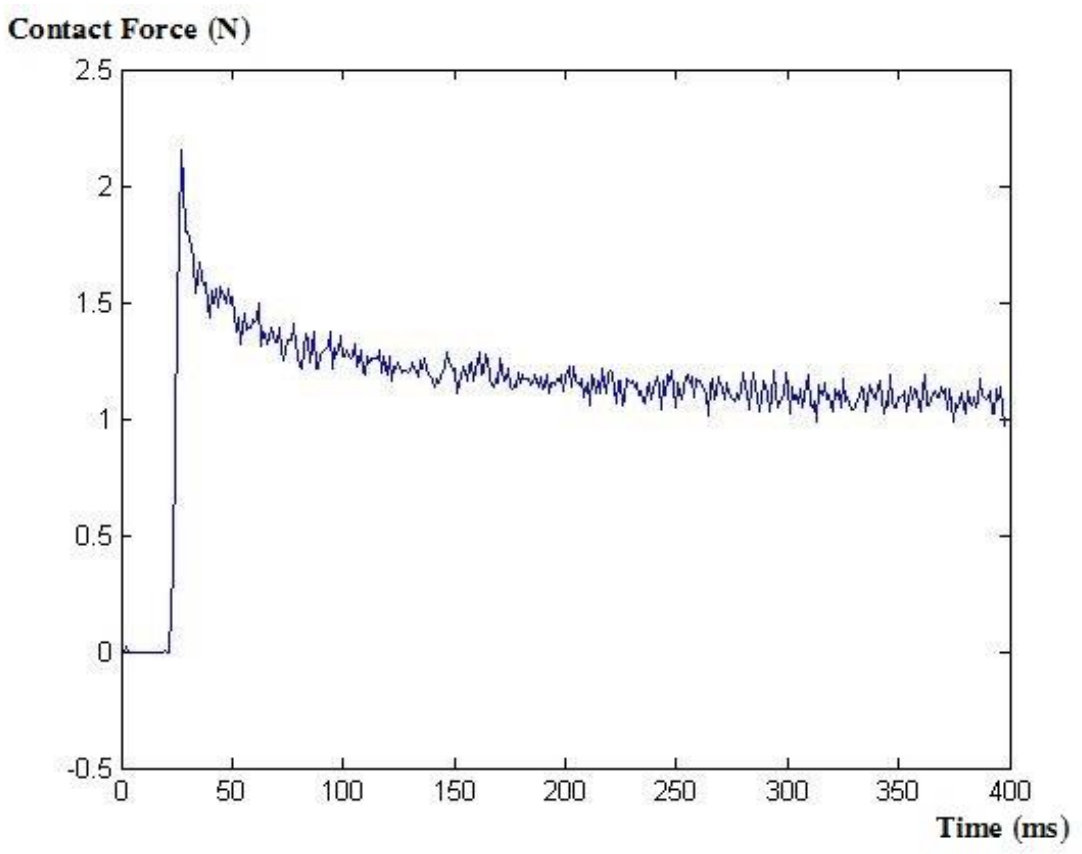

Figure 5. Contact Force Control Result

\section{Acknowledgments}

This research was supported by the Basic Science Research Program through the National Research Foundation of Korea (NRF) funded by the Ministry of Education (2017R1D1A1B03031245)

\section{References}

[1] Robert Bosch GmbH, CAN specification version 2.0, (1991).

[2] FlexRay Consortium, FlexRay protocol specification V3.0.1, (2010).

[3] S. Shaheen, D. Heffernan and G. Leen, "A gateway for time-triggered control networks", Microprocessors and Microsystems, vol. 31, no. 1, (2007), pp. 38-50.

[4] S.-H. Seo, J.-H. Kim, S. Hwang, K. H. Kwon and J. W. Jeon, "An evaluation of the FlexRay-CAN gateway-embedded system in the HEV test bench", IEEE International Symposium on Industrial Electronics, (2009), pp. 664-669.

[5] H. Li, H. Zhang, D. Peng and W. Huang, "Design and application of communication gateway based on FlexRay and CAN", International Conference on Electronic Computer Technology, (2009), pp. 664-668.

[6] E.G. Schmidt, M. Alkan, K. Schmidt, E. Yürüklü and U. Karakaya, "Performance evaluation of FlexRay/CAN networks interconnected by a gateway", International Symposium on Industrial Embedded Systems, (2010), pp. $209-212$.

[7] G. C. Walsh, H. Ye and L. G. Bushnell, "Stability analysis of networked control systems", IEEE Transactions on Control Systems Technology, vol. 10, no. 3, (2002), pp. 438-446.

[8] W. Zhang, M. S. Branicky and S. M. Phillips, "Stability of networked control systems", IEEE Control Systems, vol 21, (2001), pp. 84-99.

[9] N. Kim and C. Moon, "Actuator Control based on Interconnected Heterogeneous Networks", The Journal of The Institute of Internet, Broadcasting and Communication, vol. 17, no. 2, (2017), pp. 57-62

[10] H. Lee, I. Park, K. Lee and M. Sunwoo, "Timing analysis of FlexRay bus for gateway design", KSAE Annual Conference, (2011), pp. 133-140.

[11] R. I. Davis, A. Burns, R. J. Bril and J. J. Lukkien, "Controller area network (CAN) schedulability analysis: Refuted, revisited and revised”, Real-Time Systems, vol. 35, no. 3, (2007), pp. 239-272, 2007.

[12] Wikipedia, Integer programming, available at: https://en.wikipedia.org/wiki/Integer_programming, accessed: April 5, 2017. 


\section{Authors}

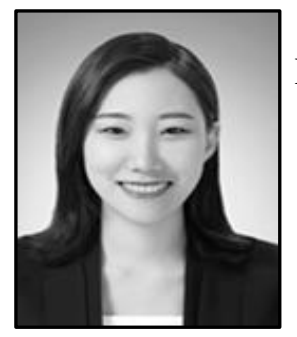

Nayeon Kim, she is a Graduate student, School of electrical Engineering, Kookmin University.

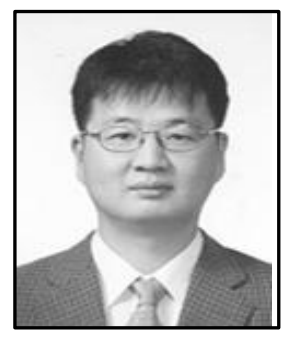

Chanwoo Moon, he is a Ph.D., Associate professor, School of electrical Engineering, Kookmin Univ. 